\title{
Patient and clinician discourses shaped the process of rehabilitation after a stroke
}

Bendz M. Rules of relevance after a stroke. Soc Sci Med 2000 Sep;51:713-23.

QUESTIONS: How do people $\leqslant 65$ years of age experience having had a stroke and deal with the rehabilitation process? How is their rehabilitation described by clinicians in medical records?

Design

Discourse analysis.

Setting

A hospital in Sweden and subsequent links in the chain of care.

\section{Patients}

6 men and 4 women $\leqslant 65$ years of age (age range $58-65$ y) who had a first stroke and spoke and understood English or Swedish.

\section{Methods}

Data sources included transcripts from patient interviews held 3-4 months after the stroke and transcripts of relevant information from the patients' medical records for the first 3 months after the stroke. Interview transcripts were coded to explore which experiences patients focused on and how they understood their experiences. Medical records were coded to explore what clinicians found relevant to report about patients and their rehabilitation activities. The analysis focused on the function and consequences of the different discourses.

\section{Main findings}

Patient experiences of stroke. Patients described themselves as being in a period of transition, in which their primary goal was to regain their former social identities. The main effect of the stroke was the dysfunction of specific parts of the body. Fatigue took command of patients' daily lives. They did not understand fatigue as something to be helped by rehabilitation activities, but felt that they had to cope with it on their own. Patients experienced a new uncertainty, fearing future strokes with more devastating consequences.

Patient understanding of the rehabilitation process. Training of impaired body parts was seen as the means to recovery. Patients trusted clinicians as experts of their disease and rehabilitation, and validated their own progress according to clinician judgments. Patients adopted a subordinate role, which made it difficult for them to later find solutions on their own.

Medical recording of patients. Medical records about patients primarily reflected the voices of physicians; those of nurses, patients, and their relatives were rare or absent. Patients were usually represented in short, abbreviated terms, as fragmented bodies with certain symptoms, impairments, and dysfunctions; their social context was essentially excluded.

Medical recording of rehabilitation process. Discourse about rehabilitation focused on risk prevention, progress or regress of symptoms, and medical treatment. Clinicians shouldered the responsibility for treatment, taking on an authoritative position. Later in the rehabilitation process, patient concerns were represented in the medical records, as their own assessments of their bodily and cognitive functions were solicited and recorded. The rehabilitation process was assessed in terms of options and obstacles, which were defined by clinicians. Patient expressed goals were sometimes presented, but no further comments or plans related to these goals were recorded. Often, problems mentioned by patients (eg, fatigue) were simply noted, without further consideration.

\section{Conclusions}

The discourses of patients who had a first stroke and their clinicians were similar in terms of their biomedical focus on physical disabilities, and their view of the clinician as authoritative and the patient as subordinate. They differed in that patients saw themselves as individuals who wanted to recapture their former social identities, whereas in the medical records, they were portrayed by clinicians as fragmented bodies of a certain age with certain impairments and dysfunction.

\section{COMMENTARY}

Bendz uses discourse analysis to explore the divergent and overlapping ways that healthcare professionals and patients construct "the stroke survivor." Discourse analysis focuses on talk and texts as social practices that create the objects they describe. There are various types of discourse analysis, ranging from apolitical description and explanation of the social world, to critical discourse analysis that analyses how relations of power and dominance are produced and reproduced in society. ${ }^{12}$

A note of caution is required when evaluating the findings of this study. Any discourse analysis is an interpretation or "reading" of the discourses in a particular context. Another analyst or a different context could produce different interpretations. Nevertheless, many of Bendz's findings resonate with observations in other discourse analyses. Heartfield also found that nursing care was invisible or portrayed as "fragmented body work" in the medical record, ${ }^{3}$ which is structured by the body systems focus of medical science. Smith noted that documentary practices both reflect the relations of power in organisations and serve to reinforce those hierarchies. ${ }^{4}$ In the process, nursing knowledge and nurses' work is erased.

Because discourse analysis is context dependent, a limitation of this study is that it was undertaken in Sweden. Possibly, patients' expectations for control over care differ between Sweden and other countries. It would be valuable to replicate the study in other geographic settings.

Discourse analysis offers nurses a way to critically analyse how their discourses are shaped by power relations and the effects these have on patients, families, and nursing practice. As such, it is another tool to achieve reflective practice. Janice McCormick, RN, PhD University of Victoria School of Nursing Lower Mainland Campus, Langara College Vancouver, British Columbia, Canada

1 Lupton D. Discourse analysis: a new methodology for understanding the ideologies of health and illness. Aust J Public Health 1992:16:145-150.

2 Van Dijk TA. Principles of critical discourse analysis. Discourse E Society 1993;4;249-83.

3 Heartfield M. Nursing documentation and nursing practice: a discourse analysis. J Adv Nurs 1996;24:98-103.

4 Smith DE. Texts, facts, and femininity: exploring the relations of ruling. London, UK: Routledge, 1990

( 